\title{
Anesthetic Management "Arnold Chiari Malformation" in the well-known Case of Cystic Fibrosis
}

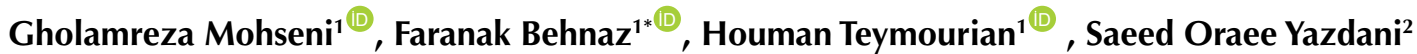 \\ ${ }^{1}$ Department of Anesthesiology, Shohada Tajrish Hospital, Shahid Beheshti University of Medical Sciences, Tehran, Iran. \\ ${ }^{2}$ Functional Neurosurgery Research Center, Shohada Tajrish Comprehensive Neurosurgical Center of Excellence, Shahid \\ Beheshti University of Medical Sciences, Tehran, Iran.
}

\begin{abstract}
Cystic fibrosis (CF) is an autosomal recessive complaint that disturbs one in 2000-3000 live births. It is a multi-organ disease and has a diversity of performances. The primary clinical structures affect the gastrointestinal and respiratory areas. Pre- and postoperative care must focus on the ideal clearance of viscous respiratory secretions. We present an anesthesia technique showed on a 24-years-old men CF case receiving general anesthesia for Arnold Chiari malformation (ACM) surgery.

Keywords: Anesthesia; Cystic Fibrosis; Arnold Chiari malformation (ACM
\end{abstract}

\author{
*Correspondence to \\ Faranak Behnaz, \\ Assistant professor of \\ Department of Anesthesiology, \\ Shohada Tajrish Hospital, \\ Shahid Beheshti University of \\ Medical Sciences, Tehran, Iran \\ Tel: +982122741174 \\ Email:faranak.behnaz@gmail.
}

com

Published online 28 September, 2020

Citation: Mohseni G, Behnaz F, Teymourian H, Oraee Yazdani S. Anesthetic Management "Arnold Chiari Malformation" in the wellknown Case of Cystic Fibrosis. Int Clin Neurosci J. 2020;7(4):216-218. doi:10.34172/icnj.2020.28.

Introduction

Cystic fibrosis (CF) is an autosomal recessive, systemic complaint that is primarily considered by chronic pulmonary infections, bronchiectasis, exocrine pancreatic insufficiency, and elevated concentration of both sodium and chloride of the sweat.1 CF happens in 2000-3000 live births, and the majority now reach adulthood.2 Respiratory findings are the most common ones for all age groups. CF is, therefore, of accumulative significance to anesthetists. Anesthetic management must be focused on the ideal clearance of sticky respiratory discharges and should diminish the risk of postoperative respiratory problems.2,3 All processes should be deliberate, but it is very significant to organize patients for operation, with daily physiotherapy, management of medicines using aerosols, and administration of vitamins and steroids if indicated. Presently, anesthesia can safely be carried out in CF patients experiencing operation, with minimal occurrence of postoperative respiratory problems. 3

\section{Case Report}

A 24-years-old man referred with a 2-months history of weakness in lower limbs. In medical history, the patient had CF and was under treatment by steroids and salbutamol. The patient was awake, well adapted to time and place. After a detailed assessment, a magnetic resonance imaging (MRI) scan patient identified to have with Arnold Chiari malformation (ACM) (Figure 1). He scheduled to the operating room for ACM operation.

Lab tests and electrocardiography results were normal. The results of the respiratory function test indicated severe obstructive lung diseases. In the posterior-anterior chest radiography, an increase in vascularity has found; in the thorax computed tomography (CT), a presence in line with chronic atelectasis and central, peripheral bronchiectasis were detected (Figure 2).

As soon as he entered the operating room, intravenous access (IV) inserted via $16 \mathrm{G}$ cannula. Monitoring has made by peripheral oximetry (SPO2), electrocardiogram (ECG), and noninvasive blood pressure. The left radial artery cannulated for measurement of invasive blood pressure after induction. Premedication with $2 \mathrm{mg}$ midazolam, fentanyl $200 \mu \mathrm{g}$, and lidocaine 70 $\mathrm{mg}$ administrated. The patient has oxygenated for 3-5 minutes. Anesthesia was induced by propofol $100 \mathrm{mg}$ IV, and muscle relaxation achieved using atracurium (50 $\mathrm{mg}$ ) IV. Endo tracheal intubation performed with a cuffed endotracheal tube $8 \mathrm{~mm}$ ID, fixation, and placement established by end-tidal carbon dioxide (ETCO2). The patient's operation was done in a prone position whiteout any related injuries due to positioning.

Anesthesia Maintenance was done with Propofol infusion $(6 \mathrm{mg} / \mathrm{kg} / \mathrm{h}), \mathrm{O} 2(100 \%)$, and Atracurium as required. During surgery, hydrocortisone (200 mg)

(C) 2020 The Author(s). This is an open access article distributed under the terms of the Creative Commons Attribution License (http:// creativecommons.org/licenses/by/4.0/), which permits unrestricted use, distribution, and reproduction in any medium, provided the original work is properly cited. 


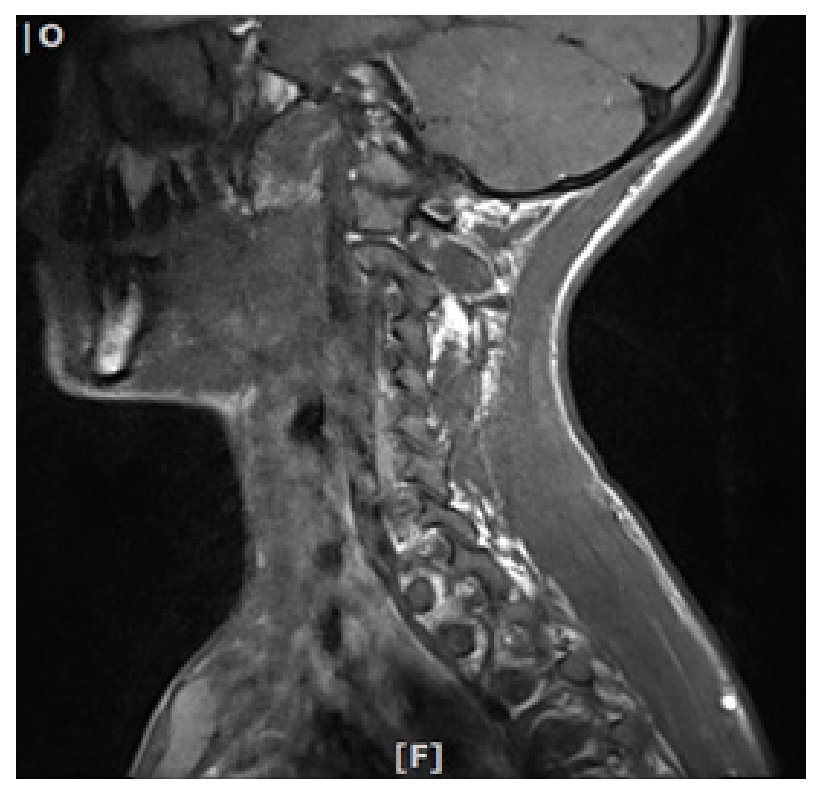

Figure 1. MRI scan patient has identified to have ACM.

administered. Controlled ventilation with a respiratory rate of $14 / \mathrm{min}$, tidal volume of $6 \mathrm{cc} / \mathrm{kg}$ preserved during operation, and the patient's condition was stable hemodynamically during the procedure. After examination of the posterior fossa and foramen magnum, posterior exclusion of the $\mathrm{C} 1$ arch completed, and the patient have held in reserve to the supine position.

After surgery, the patient was straight transmitted to the intensive care unit (ICU) and ventilated with positive pressure mode. One day later, the patient removed from mechanical ventilation. Finally, he has discharged from the hospital with a normal general situation.

\section{Discussion}

Developments in treatment now consent more than 50\% of patients with CF to reach maturity, and as these patients live longer the probability that they will be surgical entrants increase. 3

Pre-anesthetic assessment: history and clinical evaluation must make with a specific note of the most pulmonary function tests.4-6

The technique of induction: venous access should secure before any induction technique. Anticholinergic therapy should give before the induction of anesthesia. In general, anesthesia pre-oxygenation treatment can serve the patient with CF. Fluid therapy is important for these patients. Well hydration therapy can be more easily removed secretion from lungs. 6

Post-anesthetic assessment: All patients should be received 3-4 Li oxygen to breathe in the recovery room. Analgesics that do not depress the respiratory system should use in the postoperative period.6 Chest physiotherapy may also help eliminate discharges.5 specifically, in patients with hypoxemia and hypercarbia

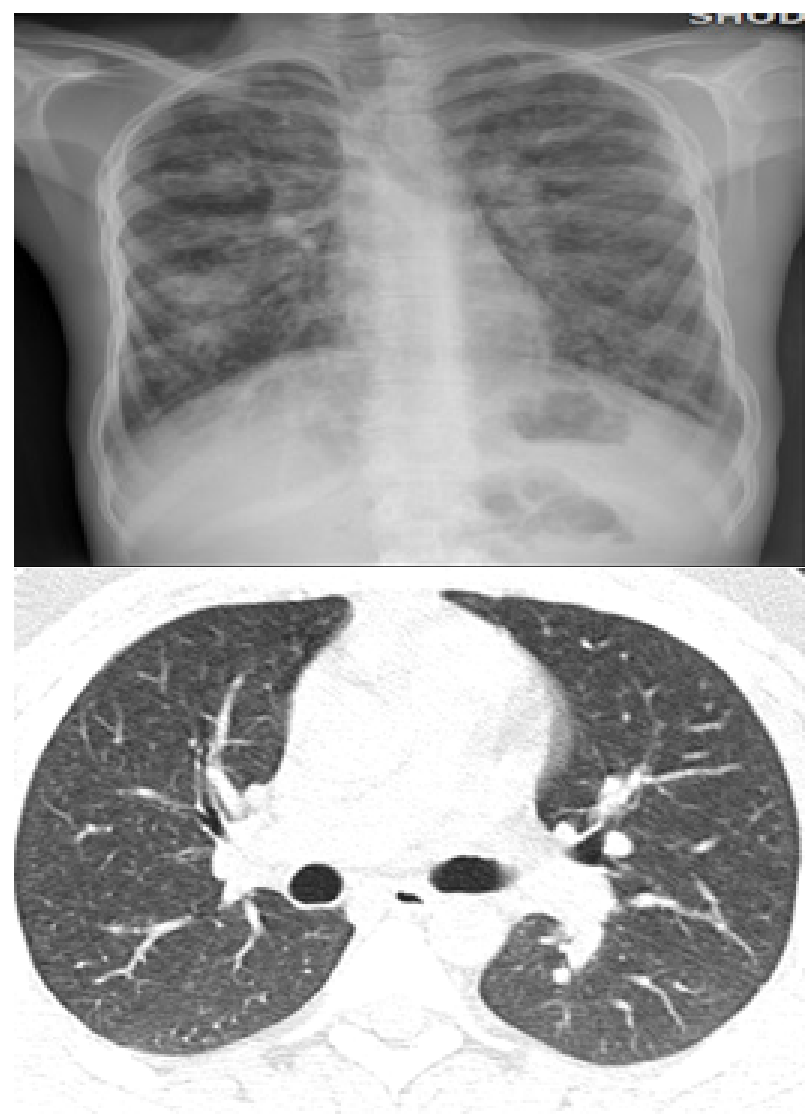

Figure 2. Chest radiography, increase in vascularity was found; (A) in the thorax $\mathrm{CT}$, a presence in line with chronic atelectasis and central, peripheral bronchiectasis were detected (B).

postoperative ventilation was needed.4

Patients with $\mathrm{CF}$ remain to be at risk for respiratory depression, such as pneumothorax, pneumonia, atelectasis, and airway obstruction. Intensive care unit may be the most suitable setting for close monitoring, continued intravenous hydration, airway management, and chest physiotherapy, after major operation. 6

In this case, we achieved a successful outcome using optimal preoperative care with associated ACM.

\section{Conclusion}

$\mathrm{CF}$ is a complex disease involving multiple organ systems; anesthetic supervision of the patient with CF may be complicated.

\section{Conflict of Interest Disclosures:}

The authors declare that they have no conflict of interest.

\section{Ethical Statement}

An educated agreement for publication of this study acquired for the patient.

\section{References}

1. Della Rocca G. Anaesthesia in patients with cystic fibrosis. Curr Opin Anaesthesiol. 2002;15(1):95-101. doi: 
10.1097/00001503-200202000-00014.

2. Höhener D, Blumenthal S, Borgeat A. Sedation and regional anaesthesia in the adult patient. Br J Anaesth. 2008;100(1):816. doi: 10.1093/bja/aem342.

3. Huffmyer JL, Littlewood KE, Nemergut EC. Perioperative management of the adult with cystic fibrosis. Anesth Analg. 2009;109(6):1949-61. doi: 10.1213/ ANE.0b013e3181b845d0.

4. Aanæs K. Bacterial sinusitis can be a focus for initial lung colonisation and chronic lung infection in patients with cystic fibrosis. J Cyst Fibros. 2013;12 Suppl 2:S1-20. doi: 10.1016/ s1569-1993(13)00150-1.

5. Cameron AJ, Skinner TA. Management of a parturient with respiratory failure secondary to cystic fibrosis. Anaesthesia. 2005;60(1):77-80. doi: 10.1111/j.1365-2044.2004.03973.x.

6. Fitzgerald M, Ryan D. Cystic fibrosis and anaesthesia. Continuing Education in Anaesthesia Critical Care \& Pain. 2011;11(6):204-9. doi: 10.1093/bjaceaccp/mkr038. 